transfer; the decision depends on the individual. A young patient with peritubal adhesions in whom fimbryolysis or salpingolysis, or both, is possible, should initially be offered tubal surgery. For a patient in her mid-30s who has extensive pelvic damage in vitro fertilisation and embryo transfer would probably be the treatment of choice.

In $30-40 \%$ of couples factors in the man are responsible for the fertility problems. The algorithm shows the investigation and management, but the success rate of most treatments other than artificial insemination by donor and in vitro fertilisation and embryo transfer is poor. These cases should be handled with particular carf as the man's ego is more vulnerable than the woman's in many situations, and suggesting that pregnancy can be achieved only by artificial insemination by donor requires tact.

A combination of factors are responsible in about $5-15 \%$ of couples with subfertility. The value of the postcoital test is controversial, but this is often the first indication that such factors are present. If the result of the postcoital test is abnormal-that is, less than five motile sperm per high power microscope field-and has been performed at the right time of the cycle-that is, the day of ovulation - the patient needs investigating further. Ovulation can be timed with the aid of the temperature chart, although results of the test will be positive in at least twice as many people if ovulation is timed by ultrasound. Antisperm antibodies should be sought in both partners. A cervical mucus sperm penetration test should be performed. Mucus from a woman of known fertility is paired with the sperm under investigation, and sperm from a man of known fertility is paired with the mucus under investigation. The results of the cross hostility test may indicate that the problem lies primarily with the cervical mucus. The concentration of antisperm antibodies may then be measured in the plasma of the woman, but this is of academic interest only as there is no effective treatment. If facilities are not available for gamete intrafallopian transfer or in vitro fertilisation and embryo transfer a trial of condom treatment may be considered. The couple are instructed to have intercourse with a condom except at midcycle. The value of condom treatment has not been proved.

If sperm fails to penetrate normal cervical mucus antisperm antibodies may be sought in both the serum and the seminal plasma of the man. Again, this is of academic interest as no treatment is of proved value. High dose steroids may be tried for three months, however, before suggesting artificial insemination by donor or in vitro fertilisation and embryo transfer.

Finally, about $20 \%$ of couples will have no obvious cause of infertility as determined by the tests detailed above. Regional centres with a particular interest in fertility may go on to perform detailed monitoring of follicular growth to try to exclude luteinised unruptured follicle syndrome and daily hormonal investigations throughout the menstrual cycle (to try to exclude the inadequate luteal phase). If these facilities are not available then the choice lies between referring patients for in vitro fertilisation and embryo transfer or gamete intrafallopian transfer and offering no further treatment other than the reassurance that about $40 \%$ of such couples will eventually conceive.

\section{Conclusions}

Infertility is a major problem that affects $10 \%$ of all couples. It imposes both psychological and psychosexual strains on relationships and should therefore be investigated and treated as rapidly as possible. The recent advances of in vitro fertilisation and embryo transfer mean that pregnancy is now a possibility for many couples. The availability of such treatment is still limited, however, particularly in the National Health Service. Patients should be reviewed regularly while undergoing treatment for infertility and given encouragement and support. After a reasonable trial of treatment patients should be given a realistic estimate of their chances of achieving a pregnancy and should be referred to adoption agencies if appropriate or counselled for childlessness.

Useful address: National Association for the Childless, 318 Summer Lane, Birmingham B19 3RL.

\section{References}

1 Stanley E. Psychosexual problems. Br Med f 1987;294:39-41.

2 Franks S. Primary and secondary amenorrhoea. BrMed $\mathcal{f}$ (in press).

\title{
For Debate...
}

\section{LMSSA: A back door entry into medicine?}

\author{
RICHARD WAKEFORD
}

In December 1982, 10 final year clinical medical students at Cambridge failed their qualifying examinations. Eight immediately chose to sit the examination of the Society of Apothecaries of London, a body that does not run a medical course but which has the right to set qualifying medical examinations under a charter granted by King James I. Seven of the students passed the four parts of the examination straight away, including both students who failed more than one subject in the Cambridge examinations. The eighth student passed the society's exam three months later.

Hughes Hall, Cambridge CB1 2EW

RICHARD WAKEFORD, MA, fellow

\section{"Second rate qualification"}

I reported these facts in the journal World Medicine, together with the suggestions that they offered prima facie evidence of a "second rate qualification" and that the General Medical Council, which is responsible for overseeing examination standards, should take some action. The General Medical Council duly wrote and asked what evidence there was for these assertions, and I repeated the story. I remarked that the council, which collects the annual examination returns from licensing bodies-universities and nonuniversity licensing bodies-was in an excellent position to collect further evidence. It could publish the figures for students who fail at their university and pass under one of the non-university licensing bodies (the Society of Apothecaries of London, the English Conjoint Board, and the Scottish Triple Qualification) and vice versa. 
The article caused little general interest at the time, but there is legitimate public concern over the notions of a "back door" entry to medicine and the possibility of "second rate qualifications." Are such assertions fair?

A perusal of the examination regulations and synopses of the society does not inspire great confidence that the procedures are particularly up to date. They refer, for example, to candidates being tested about "the taking of dying declarations" and certification "under the Lunacy Laws," which were last passed in 1890 and are now totally defunct. The language and content is quite bizarre, with talk of the duties of "medical men" and much reference to forensic medicine: this gets nine paragraphs (compared with one each for surgery, midwifery, and pathology), including "symptoms, treatment, post-mortem appearances and fatal dose of the principal mineral, organic and gaseous poisons."

\section{More bites at the cherry}

The four parts of the final examination of the Society of Apothecaries normally take place 11 times a year. The regulations of the society state that they must all be passed within two years. Even allowing that candidates cannot take the same subject for three months, this gives candidates far more "goes" than they would generally get in medical schools, some of which allow students only two attempts at finals. It also provides a less demanding "diet" of examination, as most medical schools run them only twice a year. Any examination system, no matter how it attempts to make precise judgments of candidates, has an element of chance and uncertainty in it. This means that sometimes candidates who should not have passed will do so. The Society of Apothecaries system increases this chance passing element.

The society's examiners also have a great problem caused by the sorts of candidates that they have to assess. Many are trained overseas, and many are failed students from British medical schools. As a whole group they would probably not be of a standard equivalent to a graduating class from a British medical school. Thus though the examiners may be reasonably good at putting candidates in order (first, second, third, and so on), research shows that they have difficulty in setting standards for passing, especially in circumstances like these, in which there are no explicit criteria for passing. ${ }^{2}$ Put crudely, the result is that a poor candidate is more likely to pass when he or she is examined in a group of overall low ability than when examined in a group of overall high ability.

The examiners' other problem is caused by their lack of personal knowledge of the candidates. Whatever medical schools' regulations say examiners' pass-fail decisions can be moderated by the inevitable wealth of information about their students. The society's examiners, though possessing a "certificate of good moral character" for each candidate from his or her medical school dean, are denied this. Yet the decision they have to make is a crucial one-should this student be given the privileges and responsibilities of a doctor?

The General Medical Council's principal active method of monitoring examination standards is by means of periodic inspections. The advice inspectors are given, however, is general-for example, to inspect "the standards achieved by candidates." 3 Though they can comment on the fairness, conduct, and validity of examinations, it is difficult to imagine how the inspectors are more able than the examiners to overcome the problem of setting standards.

\section{Circumstantial evidence}

The examiners, then, have an extremely difficult task. But is there any evidence, other than the eight students, to support the suggestion that these examinations are of a lower standard than those of the universities? In the absence of published differential pass rates, based on the same students' performances under both systems, the evidence is necessarily circumstantial. But it is strong. With about 4000 medical students taking their final exams each year it would be fair to expect a small proportion-perhaps $100(2-3 \%)$ - to fail to make the grade, as happens in other subjects, and not to go on to practise medicine. Yet according to the Universities' Statistical Record the official number of students leaving unqualified because they had failed their final examinations was 16 in 1984 - fewer than one per medical school (Universities' Statistical Record, personal communication). According to the General Medical Council, however, 93 medical students qtalified that same year solely by passing the Society of Apothecaries exam or that of one of its two sister nonuniversity licensing bodies. ${ }^{4}$

So the examination is almost certainly easier for a candidate from the United Kingdom than comparable university examinations in two senses-namely, more goes means a greater chance of passing, and the group of examinees as a whole is likely to be of a lower standard than a graduating class in a British medical school, thus giving a poor candidate a better chance. None of this absolutely proves the case of lower standards, of course, but it suggests strongly that, on the balance of probabilities, with the mechanism of the non-university licensing bodies, some poorer students are qualifying as doctors. And clearly, some students are qualifying who would not otherwise qualify (or who would do so only after further study).

Perhaps the oddest thing, however, is why the system exists at all. Why should qualification be possible through this method anyway? The reason generally put forward is that it offers a method for deserving foreign doctors-for example, refugees-to obtain a medical qualification and so practise in this country. This is no reason, however, to support an examination of lower standards, and no argument for medical students in the United Kingdom, having been failed by their own universities, being able to have a second go-or even, by making use of all three non-university licensing bodies, a third or fourth go. In addition, the non-university licensing bodies are by no means almost entirely examining overseas candidates-from 1978 to 1984, for example, the Society of Apothecaries awarded its qualification to 108 candidates trained overseas and 128 students trained in Britain and Ireland. ${ }^{+}$As for refugees it should surely be possible to persuade one or more British medical schools to enter the occasional refugee for its examinations.

In 1985 the General Medical Council's education committee concluded that "in view of the small numbers involved, the subsequent qualification of students who had failed the examinations of their university through the examinations of one of the nonuniversity licensing bodies did not constitute a serious problem." This is a strange argument. If there is concern that the standards of the non-university licensing bodies are lower than those of the medical schools then surely the number qualifying is immaterial?

\section{Frustrated public duty}

When British medical schools fail a student in the final examination it may simply be that the student is being referred for further study and experience, though, sadly, some students will fail absolutely. The system whereby British students can take the examinations of such bodies as the Society of Apothecaries frustrates the universities in their necessary public duty of preventing less able students from entering practice, whether temporarily or permanently. Many lay people will be startled to learn that such a mechanism exists. It is obviously a back door into medicine, is arguably a second rate qualification, and is certainly a matter for public concern.

\section{References}

1 Waketord RE. Loophole for unfit students? World Medicine 1983;29 October:14-5.

2 Wakeford RE, Roberts S. Short answer questions in an undergraduate qualifying examination: a study of examiner variability. Med Educ 1984;18:168-73.

3 Education Committee. Minutes of meeting 15 February 1984. Minutes of the General Medical Education Committee. Minu
Council 1984;cxxi:48-9.

4 Sub-Committee on the Examination Returns. Report of meeting March 1985. Minutes of the General Medical Council 1985; cxxii:243-8.

(Accepted 25 March 1987) 